ESAIM: COCV

Vol. $14, \mathrm{~N}^{\mathrm{o}} 1,2008$, pp. $148-159$

DOI: $10.1051 /$ cocv:2007050
ESAIM: Control, Optimisation and Calculus of Variations

www.esaim-cocv.org

\title{
CURL BOUNDS GRAD ON SO(3)
}

\author{
Patrizio $\mathrm{NeFF}^{1}$ And Ingo MÜNCH ${ }^{2}$
}

\begin{abstract}
Let $F^{\mathrm{P}} \in \mathrm{GL}(3)$ be the plastic deformation from the multiplicative decomposition in elasto-plasticity. We show that the geometric dislocation density tensor of Gurtin in the form $\operatorname{Curl}\left[F^{\mathrm{p}}\right]$. $\left(F^{\mathrm{P}}\right)^{T}$ applied to rotations controls the gradient in the sense that pointwise$$
\forall R \in C^{1}\left(\mathbb{R}^{3}, \mathrm{SO}(3)\right): \quad\left\|\operatorname{Curl}[R] \cdot R^{T}\right\|_{\mathbb{M}^{3 \times 3}}^{2} \geq \frac{1}{2}\|\mathrm{D} R\|_{\mathbb{R}^{27}}^{2} .
$$

This result complements rigidity results [Friesecke, James and Müller, Comme Pure Appl. Math. 55 (2002) 1461-1506; John, Comme Pure Appl. Math. 14 (1961) 391-413; Reshetnyak, Siberian Math. J. 8 (1967) 631-653)] as well as an associated linearized theorem saying that
\end{abstract}

$$
\forall A \in C^{1}\left(\mathbb{R}^{3}, \mathfrak{s o}(3)\right): \quad\|\operatorname{Curl}[A]\|_{\mathbb{M}^{3 \times 3}}^{2} \geq \frac{1}{2}\|\mathrm{D} A\|_{\mathbb{R}^{27}}^{2}=\|\nabla \operatorname{axl}[A]\|_{\mathbb{R}^{9}}^{2} .
$$

Mathematics Subject Classification. 74A35, 74E15, 74G65, 74N15, 53AXX, 53B05.

Received May 19, 2006. Revised September 5, 2006.

Published online September 21, 2007.

\section{Introduction}

We show an extension to the Lie-group $\mathrm{SO}(3)$ of proper rotations of the following result for linearized kinematics: the operator $\mathrm{Curl}_{\sharp}$ (curl arranged row wise) applied to elements of the Lie-algebra of skew-symmetric matrices $\mathfrak{s o}(3)$ already controls all partial derivatives of these matrices. While in general, the operator Curl $\sharp$ cannot control the full gradient since $\mathrm{Curl}_{\sharp}$ has 9 independent entries but Grad $=\mathrm{D}$ has 27 independent entries, it does so on $\mathfrak{s o}(3)$, since they have only 3 independent components such that taking Grad gives 9 independent entries making the relation between $\mathrm{Curl}_{\sharp}$ and Grad invertible.

Such a result can at least be traced back implicitly to Nye [26], who investigated infinitesimal rotations of the crystal lattice due to dislocation motion $[11,19,22,23]$. He showed for small plastic deformations and zero elastic strains that

$$
-\operatorname{Curl}_{\sharp}\left[\operatorname{skew}\left[\varepsilon^{\mathrm{p}}\right]\right]=\left(\nabla \operatorname{axl}\left[\operatorname{skew}\left[\varepsilon^{\mathrm{p}}\right]\right]\right)^{T}-\operatorname{tr}\left[\left(\nabla \operatorname{axl}\left[\operatorname{skew}\left[\varepsilon^{\mathrm{p}}\right]\right]\right)^{T}\right] \mathbb{1},
$$

\footnotetext{
Keywords and phrases. Rotations, polar-materials, microstructure, dislocation density, rigidity, differential geometry, structured continua.

1 Department of Mathematics, Technische Universität Darmstadt, Schlossgartenstrasse 7, 64289 Darmstadt, Germany; neff@mathematik.tu-darmstadt.de

${ }^{2}$ Institut für Baustatik, Universität Karlsruhe (TH), Kaiserstrasse 12, 76131 Karlsruhe, Germany; im@bs.uka.de

(c) EDP Sciences, SMAI 2007
} 
where $\varepsilon^{\mathrm{p}} \in C^{1}\left(\Omega, \mathbb{M}^{3 \times 3}\right)$ is the non-symmetrical infinitesimal plastic distortion with $\Omega \subset \mathbb{R}^{3}$ the reference configuration. Here, for second order tensors skew $[X]:=\frac{1}{2}\left(X-X^{T}\right)$, 1 is the identity tensor, $\|X\|^{2}=\sum_{i} X_{i}^{2}$, $\operatorname{tr}[X]$ the trace, the axial vector $\operatorname{axl}[A]$ is defined such that $A \cdot v=\operatorname{axl}[A] \times v$ for all $A \in \mathfrak{s o}(3)$ and $v \in \mathbb{R}^{3}$, see also (2.6) and $\nabla \varphi$ is the Jacobian-matrix. With $A \cdot B$ we denote simple contraction, with $A: B$ double contraction. See Section 4 for the proof and background of (1.1). Abbreviating $A=\operatorname{skew}\left[\varepsilon^{\mathrm{P}}\right] \in C^{1}(\Omega, \mathfrak{s o}(3))$ one deduces

$$
\begin{aligned}
-\operatorname{Curl}_{\sharp}[A] & =(\nabla \operatorname{axl}[A])^{T}-\operatorname{tr}\left[(\nabla \operatorname{axl}[A])^{T}\right] 11 \Leftrightarrow \\
\nabla \operatorname{axl}[A] & =-\left(\operatorname{Curl}_{\sharp}[A]\right)^{T}+\frac{1}{2} \operatorname{tr}\left[\left(\operatorname{Curl}_{\sharp}[A]\right)^{T}\right] 11,
\end{aligned}
$$

which leads to, $c f .(4.9)$

$$
\forall A \in C^{1}\left(\mathbb{R}^{3}, \mathfrak{s o}(3)\right): \quad\left\|\operatorname{Curl}_{\sharp}[A]\right\|_{\mathbb{M}^{3 \times 3}}^{2} \geq \frac{1}{2}\|\mathrm{D} A\|_{\mathbb{R}^{27}}^{2}=\|\nabla \operatorname{axl}[A]\|_{\mathbb{R}^{9}}^{2},
$$

in turn implying infinitesimal rigidity (1.7). Recall also the definition of the curl of displacements $u \in C^{1}\left(\Omega, \mathbb{R}^{3}\right)$ and the relation to the infinitesimal rotations skew $[\nabla u]$,

$$
\operatorname{curl}[u]:=\nabla \times u=2 \operatorname{axl}[\operatorname{skew}[\nabla u]] .
$$

The modern theory of finite plasticity is based on the Kröner, Lee, Kondo, Bilby $[2,14,17,20]$ multiplicative decomposition $F=F^{\mathrm{e}} \cdot F^{\mathrm{p}}$ of the deformation gradient $F=\nabla \varphi$ into structural elastic and plastic components. In single crystal plasticity $F^{\mathrm{p}}$ represents the deformation solely resulting from the formation of defects such as dislocations while $F^{\mathrm{e}}$ is due to elastic stretch and elastic rotation of the lattice. In general, $F^{\mathrm{e}}$ and $F^{\mathrm{p}}$ have not the form of a Jacobian matrix, they are incompatible, i.e. $\operatorname{Curl}_{\sharp}\left[F^{\mathrm{e}}\right], \operatorname{Curl}_{\sharp}\left[F^{\mathrm{p}}\right] \neq 0$, a property related to the formation of dislocations. The most general stored defect energy, measuring the incompatibility in $F^{\mathrm{p}}$, which is invariant under a compatible change in the reference configuration $[4,21,29,31]$ is expressible in the geometrical dislocation density tensor $G=\frac{1}{\operatorname{det}\left[F^{\mathrm{p}} \mathrm{Cur}\right.} \operatorname{Cur}_{\sharp}\left[F^{\mathrm{p}}\right] \cdot\left(F^{\mathrm{p}}\right)^{T}$ which, for $R \in \mathrm{SO}(3)$, reduces to $G=\mathrm{Curl}_{\sharp}[R] \cdot R^{T}$. For the necessary background and more references on dislocations, plasticity and microstructures we refer to $[1,4,5,27,28]$.

Another motivation comes from rigidity results $[12,30]$ in the spirit of Liouville-type theorems, saying that if the gradient of a deformation is locally a rotation it must be a constant rotation together with a constant translation or more precisely

$$
\begin{aligned}
\varphi \in W^{1, \infty}\left(\Omega, \mathbb{R}^{n}\right), \quad \nabla \varphi(x) & \in \mathrm{SO}(n) \text { a.e } \Rightarrow \\
\nabla \varphi & =R=\text { const. } \Leftrightarrow \varphi(x)=R \cdot x+b .
\end{aligned}
$$

A quantized version of this fact has been given recently in [8]. They show that for bounded $\Omega \subset \mathbb{R}^{3}$ with Lipschitz boundary and $\varphi \in W^{1,2}\left(\Omega, \mathbb{R}^{3}\right)$ there exists a positive constant $C(\Omega)$ and a constant rotation $R$ such that

$$
\int_{\Omega}\|\nabla \varphi-R\|^{2} \mathrm{dx} \leq C(\Omega) \int_{\Omega} \operatorname{dist}^{2}(\nabla \varphi, \mathrm{SO}(3)) \mathrm{dx} .
$$

The respective infinitesimal rigidity result is standard in the treatment of linear elasticity and Korn's inequality, e.g. [24]. It amounts to

$$
\begin{array}{r}
u \in W^{1,2}\left(\Omega, \mathbb{R}^{3}\right), \quad \nabla u(x)+\nabla u(x)^{T}=0 \Leftrightarrow \nabla u(x) \in \mathfrak{s o}(3) \Leftrightarrow \\
\nabla u(x)=A=\text { const. } \Leftrightarrow u(x)=A \cdot x+b,
\end{array}
$$


where $A \in \mathfrak{s o}(3)$ and $b \in \mathbb{R}^{3}$ are constant. Since from $\operatorname{sym}[\nabla u(x)]=0$ it follows $\nabla u(x)=A(x) \in \mathfrak{s o}(3)$ the result (1.7) would follow by applying $\mathrm{Curl}_{\sharp}$ on both sides and using that $\mathrm{Curl}_{\sharp}$ bounds D $A$ on $\mathfrak{s o}(3)$ due to $(1.3)$. As a consequence of (1.5) it is known that for smooth, simply connected domains $\Omega \subset \mathbb{R}^{3}$ and $R \in C^{1}(\Omega, \mathrm{SO}(3))$

$$
0=\operatorname{Curl}_{\sharp}[R(x)] \Leftrightarrow R=\nabla \varphi \in \mathrm{SO}(3)=\text { const. } \Leftrightarrow \mathrm{D} R=0,
$$

thus showing that $\operatorname{Curl}_{\sharp}[R]=0 \Leftrightarrow \mathrm{D} R=0$. Obviously, $\left\|\operatorname{Curl}_{\sharp}[R]\right\|_{\mathbb{M}^{3 \times 3}}^{2} \leq 2\|\mathrm{D} R\|_{\mathbb{R}^{27}}^{2}$ by Young's inequality for all $R \in \mathbb{M}^{3 \times 3}$. The precise relation between $\operatorname{Curl}_{\sharp}$ and $\mathrm{Grad}=\mathrm{D}$ on $\mathrm{SO}(2)$ is easily understood in terms of the representation with one rotation angle $\vartheta: \Omega \subset \mathbb{R}^{2} \mapsto \mathbb{R}$

$$
R(x, y)=\left(\begin{array}{cc}
\cos \vartheta(x, y) & \sin \vartheta(x, y) \\
-\sin \vartheta(x, y) & \cos \vartheta(x, y)
\end{array}\right) \in \mathrm{SO}(2)
$$

One checks that

$$
\begin{aligned}
\left\|\operatorname{Curl}_{\sharp}[R(x, y)]\right\|_{\mathbb{R}^{2}}^{2} & =\left((\cos \vartheta)_{y}-(\sin \vartheta)_{x}\right)^{2}+\left((-\sin \vartheta)_{y}-(\cos \vartheta)_{x}\right)^{2} \\
& =\|\nabla \vartheta(x, y)\|_{\mathbb{R}^{2}}^{2}=\frac{1}{2}\|\mathrm{D} R\|_{\mathbb{R}^{8}}^{2},
\end{aligned}
$$

which led us to surmise that for three-space dimensions

$$
\exists c^{+}>0 \quad \forall R \in C^{1}\left(\mathbb{R}^{3}, \mathrm{SO}(3)\right): \quad\left\|\mathrm{Curl}_{\sharp}[R]\right\|_{\mathbb{M}^{3 \times 3}}^{2} \geq c^{+}\|\mathrm{D} R\|_{\mathbb{R}^{27}}^{2} .
$$

This is what we will prove in this note with $c=\frac{1}{2}$. In terms of the geometrical dislocation density tensor $G=\operatorname{Curl}_{\sharp}[R] \cdot R^{T}$ we observe that $\left\|\operatorname{Curl}_{\sharp}[R]\right\|_{\mathbb{M}^{3 \times 3}}^{2}=\left\|\operatorname{Curl}_{\sharp}[R] \cdot R^{T}\right\|_{\mathbb{M}^{3 \times 3}}^{2}$ by the invariance of the euclidean norm under $\mathrm{SO}(3)$. The non-trivial implication in (1.5) is a simple consequence of (1.11).

It may be that this result is known to experts in the theory of differential geometry. However, we have been unable to find a reference for it and therefore provide a direct proof herein. Let us sketch our method of proof: we use two times that for orthogonal matrices $R^{T} \cdot R=11$. The first time we take partial derivatives in fixed $j$-direction and conclude that

$$
\begin{gathered}
0=\partial_{j}[11]=\partial_{j}\left[R^{T} \cdot R\right]=\left(\partial_{j} R\right)^{T} \cdot R+R^{T} \cdot \partial_{j} R \Rightarrow \\
\left(R^{T} \cdot \partial_{j} R\right) \in \mathfrak{s o}(3), j=1,2,3,
\end{gathered}
$$

giving rise to a second order curvature measure $\widehat{\mathfrak{K}} \in \mathbb{M}^{3 \times 3}$ with nine independent components, while the second time we apply the operator $\mathrm{Curl}_{\sharp}$ :

$$
0=\operatorname{Curl}_{\sharp}\left[R^{T} \cdot R\right] \Rightarrow 0=L_{R}(\mathrm{D} R)+R^{T} \cdot \operatorname{Curl}_{\sharp}[R],
$$

relating the $\operatorname{Curl}_{\sharp}[R]$ with nine independent entries to the full gradient DR. Carefully combining both results establishes the claim.

\section{Preliminary Definitions}

Let us introduce two different arrangements of the operator Curl on second order tensors $\mathbb{M}^{3 \times 3}$. Let $X \in$ $C^{1}\left(\Omega, \mathbb{M}^{3 \times 3}\right)$ with $X_{1}, X_{2}, X_{3}$ the rows of $X$. Then, for the first arrangement, Curl $_{\sharp}$ is defined row wise as in $[21,31]$ such that

$$
\operatorname{Curl}_{\sharp}\left[X\left(x_{1}, x_{2}, x_{3}\right)\right]=\left(\begin{array}{l}
\operatorname{curl}\left[X_{1}\left(x_{1}, x_{2}, x_{3}\right)\right]-- \\
\operatorname{curl}\left[X_{2}\left(x_{1}, x_{2}, x_{3}\right)\right]-- \\
\operatorname{curl}\left[X_{3}\left(x_{1}, x_{2}, x_{3}\right)\right]--
\end{array}\right) \in \mathbb{M}^{3 \times 3} .
$$


The second arrangement is defined through $\operatorname{Curl}_{b}[X]:=\left(\operatorname{Curl}_{\sharp}[X]\right)^{T}$ and corresponds to Gurtins definition $[9,10]$ of the Curl-operator on matrices. For $X, Y \in C^{1}\left(\Omega, \mathbb{M}^{3 \times 3}\right)$ it holds

$$
\operatorname{Curl}_{\sharp}[X \cdot Y]=L_{Y}(\mathrm{D} X)+X \cdot \mathrm{Curl}_{\sharp}[Y] \in \mathbb{M}^{3 \times 3},
$$

where $L_{Y}: \mathbb{R}^{27} \mapsto \mathbb{M}^{3 \times 3}$ is a linear mapping at given $Y$, arranging all first partial derivatives in $\mathrm{D} X$ of $X$ in the correct way [25]. Let us apply $(2.2)$ to $R^{T} \cdot R=11$. We get

$$
0=\operatorname{Curl}_{\sharp}[\mathbb{1}]=\operatorname{Curl}_{\sharp}\left[R^{T} \cdot R\right]=L_{R}\left(\mathrm{D}\left(R^{T}\right)\right)+R^{T} \cdot \operatorname{Curl}_{\sharp}[R]=\tilde{L}_{R}(\mathrm{D} R)+R^{T} \cdot \operatorname{Curl}_{\sharp}[R],
$$

with $\tilde{L}_{R}: \mathbb{R}^{27} \mapsto \mathbb{M}^{3 \times 3}$, linear in $\mathrm{D} R$. Hence

$$
R^{T} \cdot \mathrm{Cur}_{\sharp}[R]=-\tilde{L}_{R}(\mathrm{D} R) .
$$

We need also to introduce the canonical identification of $\mathbb{R}^{3}$ with $\mathfrak{s o}(3)$. For

$$
A=\left(\begin{array}{ccc}
0 & -a_{3} & a_{2} \\
a_{3} & 0 & -a_{1} \\
-a_{2} & a_{1} & 0
\end{array}\right) \in \mathfrak{s o}(3)
$$

we define axl : $\mathfrak{s o}(3) \mapsto \mathbb{R}^{3}$ and anti $: \mathbb{R}^{3} \mapsto \mathfrak{s o}(3)$ through

$$
\begin{aligned}
& \operatorname{axl}\left(\begin{array}{ccc}
0 & -a_{3} & a_{2} \\
a_{3} & 0 & -a_{1} \\
-a_{2} & a_{1} & 0
\end{array}\right):=\left(\begin{array}{l}
a_{1} \\
a_{2} \\
a_{3}
\end{array}\right), \quad A_{i j}=\sum_{k=1}^{3}-\epsilon_{i j k}(\operatorname{axl}[A])_{k}=: \operatorname{anti}(\operatorname{axl}[A])_{i j}, \\
& A \cdot v=\operatorname{axl}[A] \times v, \quad \forall v \in \mathbb{R}^{3}, \quad(\operatorname{axl}[A])_{k}=\sum_{i, j=1}^{3}-\frac{1}{2} \epsilon_{i j k} A_{i j},
\end{aligned}
$$

where $\epsilon_{i j k}$ is the totally antisymmetric third order permutation tensor

$$
\epsilon_{i j k}= \begin{cases}+1 & \text { even permutation } \\ 0 & \text { two identical indices } \\ -1 & \text { odd permutation }\end{cases}
$$

Observe that for the corresponding euclidean vector- and matrix-norms one has for $A \in \mathfrak{s o}(3) \subset \mathbb{M}^{3 \times 3}$

$$
2\|\operatorname{axl}[A]\|_{\mathbb{R}^{3}}^{2}=\|A\|_{\mathbb{M}^{3 \times 3}}^{2}, \quad\|\mathrm{D} A\|_{\mathbb{M}^{3 \times 3}}^{2}=2\|\nabla \operatorname{axl}[A]\|_{\mathbb{R}^{9}}^{2} .
$$

Let us now take partial derivatives of $R^{T} \cdot R=11$. This leads to, $c f$. (1.12)

$$
R^{T} \cdot \partial_{j} R \in \mathfrak{s o}(3), \quad j=1,2,3,
$$

and we may look at the axial representation $\mathbf{k}^{j}$

$$
\mathbf{k}^{j}=\operatorname{axl}\left[R^{T} \cdot \partial_{j} R\right] \in \mathbb{R}^{3}, \quad j=1,2,3,
$$

which defines the second order curvature tensor

$$
\widehat{\mathfrak{K}}=-\left(\mathbf{k}^{1}\left|\mathbf{k}^{2}\right| \mathbf{k}^{3}\right) \in \mathbb{M}^{3 \times 3},
$$


with $\mathbf{k}^{j}$ arranged in columns. Note also that

$$
\begin{aligned}
\|\widehat{\mathfrak{K}}\|_{\mathbb{M}^{3 \times 3}}^{2} & =\sum_{i=1}^{3}\left\|\mathbf{k}^{i}\right\|_{\mathbb{R}^{3}}^{2}=\sum_{i=1}^{3}\left\|\operatorname{axl}\left[R^{T} \cdot \partial_{i} R\right]\right\|_{\mathbb{R}^{3}}^{2}=\sum_{i=1}^{3} \frac{1}{2}\left\|R^{T} \cdot \partial_{i} R\right\|_{\mathbb{M}^{3 \times 3}}^{2} \\
& =\frac{1}{2} \sum_{i=1}^{3}\left\|\partial_{i} R\right\|_{\mathbb{M}^{3 \times 3}}^{2}=\frac{1}{2}\|\mathrm{D} R\|_{\mathbb{R}^{27}}^{2} .
\end{aligned}
$$

It is basic to reconstruct all partial derivatives in $\mathrm{D} R$ from $\widehat{\mathfrak{K}}$, provided that $R$ is known. To see this, write for $i=1,2,3$

$$
[\mathrm{D} R]_{i}:=\partial_{i} R=R \cdot R^{T} \cdot \partial_{i} R=R \cdot \operatorname{anti}\left(-\operatorname{axl}\left[R^{T} \cdot \partial_{i} R\right]\right)=-R \cdot \operatorname{anti}\left(\mathbf{k}^{i}\right) \in \mathbb{M}^{3 \times 3},
$$

hence, $\mathrm{D} R \in \mathbb{R}^{27}$ may be reconstructed with the help of a mapping $Z_{R}: \mathbb{M}^{3 \times 3} \mapsto \mathbb{R}^{27}$, linear at given $R \in \mathrm{SO}(3)$, with

$$
Z_{R}(\widehat{\mathfrak{K}})=\mathrm{D} R \text {. }
$$

Inserting this relation into (2.4) we obtain

$$
R^{T} \cdot \operatorname{Curl}_{\sharp}[R]=-\tilde{L}_{R}\left(Z_{R}(\widehat{\mathfrak{K}})\right) .
$$

Observe that the composition mapping $\tilde{L}_{R} \cdot Z_{R}: \mathbb{M}^{3 \times 3} \mapsto \mathbb{M}^{3 \times 3}$ is again linear at given $R$. If we can show that $\tilde{L}_{R} . Z_{R}$ is invertible for given $R$, then from $(2.15)$, we can uniquely express the Curl $_{\sharp}$ in terms of the second order curvature tensor $\widehat{\mathfrak{K}}$

$$
\left[-\tilde{L}_{R} \cdot Z_{R}\right]^{-1}\left[R^{T} \cdot \operatorname{Curl}_{\sharp}[R]\right]=\widehat{\mathfrak{K}} .
$$

\section{Detailed COMpUtation in Index nOtation}

We will show now that the composition mapping $\tilde{L}_{R} \cdot Z_{R}$ in (2.16) is indeed invertible for given $R$. In order to do so we switch to index notation and use $\mathrm{Curl}_{b}$ instead of $\mathrm{Curl}_{\sharp}$ (see (2.1)) without compromising the result. Summation over repeated indices is understood and we use orthogonal basis elements making the distinction between co- and contravariant bases obsolete. In this section we distinguish also between symbolic (bold) and component notation of tensors, e.g. $\mathbf{R}=R_{a b} \mathbf{e}_{a} \otimes \mathbf{e}_{b}$. Since $\operatorname{Grad}[\mathbf{R}]=\mathrm{DR}$ is a third order tensor, while $\operatorname{Curl}_{b}[\mathbf{R}]$ is a second order tensor we will use first the orthogonality relation $\mathbf{R}^{T} \mathbf{R}=\mathbb{1 1}$ for rotations in order to map the third order gradient tensor into a second order curvature tensor $\widehat{\mathfrak{K}}$, as already alluded too above.

\subsection{The gradient of a rotation and the 2 nd order curvature}

By taking the partial derivatives in fixed $j$-direction of the orthogonality relation $\mathbf{R}^{T} \cdot \mathbf{R}=11$ and observing (1.12) we obtained the three $(j=1,2,3)$ second order antisymmetric tensors

$$
\mathbf{K}^{j}:=\mathbf{R}^{T} \mathbf{R}_{, j}, \quad \mathbf{K}^{j} \in \mathfrak{s o}(3) \subset \mathbb{M}^{3 \times 3} .
$$

The corresponding axial vectors $\mathbf{k}^{j}:=\operatorname{axl}\left[\mathbf{K}^{j}\right] \in \mathbb{R}^{3}$ have been assembled together in the second order curvature tensor $\widehat{\mathfrak{K}}$

$$
\begin{aligned}
\widehat{\mathfrak{K}} & :=-\left(\mathbf{k}^{1}\left|\mathbf{k}^{2}\right| \mathbf{k}^{3}\right) \\
& =-\mathbf{k}^{j} \otimes \mathbf{e}_{j}=-\operatorname{axl}\left[\mathbf{K}^{j}\right] \otimes \mathbf{e}_{j}=-\operatorname{axl}\left[\mathbf{R}^{T} \cdot \mathbf{R}_{, j}\right] \otimes \mathbf{e}_{j}=\frac{1}{2}\left(\boldsymbol{\epsilon}:\left(\mathbf{R}^{T} \cdot \mathbf{R}_{, j}\right)\right) \otimes \mathbf{e}_{j}
\end{aligned}
$$


In (3.2) we may perceive the mapping between $\widehat{\mathfrak{K}}$ and the full gradient of $\mathbf{R}$, which can be seen now more clearly again in index notation

$$
\widehat{\mathfrak{K}}:=\frac{1}{2} \epsilon_{i c b} R_{a c} R_{a b, j} \mathbf{e}_{i} \otimes \mathbf{e}_{j}
$$

The linear map connecting the full gradient $\operatorname{Grad}[\mathbf{R}]$ with the second order curvature tensor $\widehat{\mathfrak{K}}$ reads

$$
\operatorname{Grad}[\mathbf{R}]:=R_{a b, j} \mathbf{e}_{a} \otimes \mathbf{e}_{b} \otimes \mathbf{e}_{j}=\epsilon_{b i k} R_{a k} \widehat{\mathfrak{K}}_{i j} \mathbf{e}_{a} \otimes \mathbf{e}_{b} \otimes \mathbf{e}_{j},
$$

and by defining

$$
\widehat{Z}_{R}:=\epsilon_{b i k} R_{a k} \mathbf{e}_{a} \otimes \mathbf{e}_{b} \otimes \mathbf{e}_{i}=R_{a k} \epsilon_{k b i} \mathbf{e}_{a} \otimes \mathbf{e}_{b} \otimes \mathbf{e}_{i}=\mathbf{R} \cdot \boldsymbol{\epsilon}
$$

it can also be rephrased in symbolic notation:

$$
\operatorname{Grad}[\mathbf{R}]=Z_{R}(\widehat{\mathfrak{K}})=\widehat{Z}_{R} \cdot \widehat{\mathfrak{K}}
$$

where the dot denotes simple contraction. By inserting (3.3) into (3.4) we verify the expression for $\widehat{Z}_{R}$

$$
\begin{aligned}
\operatorname{Grad}[\mathbf{R}] & =\epsilon_{b i k} R_{a k} \widehat{\mathfrak{K}}_{i j} \mathbf{e}_{a} \otimes \mathbf{e}_{b} \otimes \mathbf{e}_{j}=\epsilon_{b i k} R_{a k}\left(\frac{1}{2} \epsilon_{i c d} R_{f c} R_{f d, j}\right) \mathbf{e}_{a} \otimes \mathbf{e}_{b} \otimes \mathbf{e}_{j} \\
& =\frac{1}{2}\left(\delta_{k c} \delta_{b d}-\delta_{k d} \delta_{b c}\right) R_{a k} R_{f c} R_{f d, j} \mathbf{e}_{a} \otimes \mathbf{e}_{b} \otimes \mathbf{e}_{j} \\
& =\frac{1}{2}(R_{f k} R_{f b, j}-\underbrace{R_{f b} R_{f k, j}}_{-R_{f k} R_{f b, j}}) R_{a k} \mathbf{e}_{a} \otimes \mathbf{e}_{b} \otimes \mathbf{e}_{j} \\
& =\underbrace{R_{a k}\left(R^{T}\right)_{k f}}_{\delta_{a f}} R_{f b, j} \mathbf{e}_{a} \otimes \mathbf{e}_{b} \otimes \mathbf{e}_{j}=R_{a b, j} \mathbf{e}_{a} \otimes \mathbf{e}_{b} \otimes \mathbf{e}_{j} .
\end{aligned}
$$

\subsection{The Curl of the rotation field}

As said above, we use the operator $\mathrm{Curl}_{b}$ in this part. To present its definition in index notation we write for $\mathbf{X} \in C^{1}\left(\Omega, \mathbb{M}^{3 \times 3}\right)$

$$
\begin{aligned}
\operatorname{Curl}_{b}[\mathbf{X}] & =\left(\partial_{a} \mathbf{e}_{a}\right) \times\left(X_{i j} \mathbf{e}_{i} \otimes \mathbf{e}_{j}\right)^{T}=\left(\partial_{a} \mathbf{e}_{a}\right) \times\left(X_{j i} \mathbf{e}_{i} \otimes \mathbf{e}_{j}\right)=\partial_{a} \epsilon_{a i k} X_{j i} \mathbf{e}_{k} \otimes \mathbf{e}_{j} \\
& =\left(\nabla \times\left(\mathbf{X}^{T} \cdot \mathbf{e}_{1}\right)\left|\nabla \times\left(\mathbf{X}^{T} \cdot \mathbf{e}_{2}\right)\right| \nabla \times\left(\mathbf{X}^{T} \cdot \mathbf{e}_{3}\right)\right)=\left(\operatorname{Curl}_{\sharp}[\mathbf{X}]\right)^{T} .
\end{aligned}
$$

Applying the operator $\mathrm{Curl}_{b}$ on the orthogonality relation $\mathbf{R}^{T} \cdot \mathbf{R}=\mathbb{1}$ we obtain

$$
\begin{aligned}
& \mathbf{0}=\operatorname{Curl}_{b}[11]=\operatorname{Curl}_{b}\left[\mathbf{R}^{T} \cdot \mathbf{R}\right]=\nabla \times\left[\left(\mathbf{R}^{T} \cdot \mathbf{R}\right)^{T}\right]=\nabla \times\left(\mathbf{R}^{T} \cdot \mathbf{R}\right)=\partial_{m} \epsilon_{m a b}\left(\mathbf{R}^{T} \cdot \mathbf{R}\right)_{a c} \mathbf{e}_{b} \otimes \mathbf{e}_{c} \\
& \quad=\epsilon_{m a b} \partial_{m}\left(\left(R^{T}\right)_{a f} R_{f c}\right) \mathbf{e}_{b} \otimes \mathbf{e}_{c}\left(\epsilon_{m a b}\left(R^{T}\right)_{a f, m} R_{f c}+\epsilon_{m a b} R_{f a} R_{f c, m}\right) \mathbf{e}_{b} \otimes \mathbf{e}_{c} \Rightarrow \\
& \operatorname{Curl}_{b}[\mathbf{R}] \cdot \mathbf{R}=\left(\nabla \times \mathbf{R}^{T}\right) \cdot \mathbf{R}=-\epsilon_{m a b} R_{f a} R_{f c, m} \mathbf{e}_{b} \otimes \mathbf{e}_{c}=L_{R}(\operatorname{Grad}[\mathbf{R}])
\end{aligned}
$$

The linear map $L_{R}$ defined implicitly in (3.9) would need an extended symbolic notation with which we can, fortunately enough, dispens. The precise definition in index notation

$$
L_{R}(\operatorname{Grad}[\mathbf{R}]):=-\epsilon_{m a b} R_{f a} R_{f c, m} \mathbf{e}_{b} \otimes \mathbf{e}_{c}=\epsilon_{m b a} R_{f a}(\operatorname{Grad}[\mathbf{R}])_{f c, m} \mathbf{e}_{b} \otimes \mathbf{e}_{c}
$$

will prove to be useful below. 


\subsection{Combination of the results}

Equation (3.9) yields the relation between the Curl (left hand side) and the full gradient of $\mathbf{R}$ (right hand side). As discussed in Section 3.1, the gradient is completely described by the second order curvature tensor $\widehat{\mathfrak{K}}$. Inserting (3.6) in (3.10) we obtain

$$
\begin{aligned}
\operatorname{Curl}_{b}[\mathbf{R}] \cdot \mathbf{R} & =L_{R}(\operatorname{Grad}[\mathbf{R}]) \\
& =\epsilon_{m b a} R_{f a}\left(Z_{R}(\widehat{\mathfrak{K}})\right)_{f c, m} \mathbf{e}_{b} \otimes \mathbf{e}_{c}=\epsilon_{m b a} R_{f a}\left(\widehat{Z}_{R} \cdot \widehat{\mathfrak{K}}\right)_{f c, m} \mathbf{e}_{b} \otimes \mathbf{e}_{c} \\
& =\epsilon_{m b a} R_{f a}\left(\epsilon_{c i k} R_{f k} \widehat{\mathfrak{K}}_{i m}\right) \mathbf{e}_{b} \otimes \mathbf{e}_{c}=\epsilon_{m b a} \epsilon_{c i k} \underbrace{\left(R^{T}\right)_{a f} R_{f k}}_{\delta_{a k}} \widehat{\mathfrak{K}}_{i m} \mathbf{e}_{b} \otimes \mathbf{e}_{c} \\
& =\epsilon_{m b a} \epsilon_{c i a} \widehat{\mathfrak{K}}_{i m} \mathbf{e}_{b} \otimes \mathbf{e}_{c}=\left(\delta_{m c} \delta_{b i}-\delta_{m i} \delta_{b c}\right) \widehat{\mathfrak{K}}_{i m} \mathbf{e}_{b} \otimes \mathbf{e}_{c} \\
& =\left(\widehat{\mathfrak{K}}_{b c}-\delta_{i m} \widehat{\mathfrak{K}}_{i m} \delta_{b c}\right) \mathbf{e}_{b} \otimes \mathbf{e}_{c}=\widehat{\mathfrak{K}}-\operatorname{tr}[\widehat{\mathfrak{K}}] \mathbb{1} .
\end{aligned}
$$

In view of the invertibility of the right hand side $(4.4)_{1}$, we continue by writing

$$
\widehat{\mathfrak{K}}=\operatorname{Curl}_{b}[\mathbf{R}] \cdot \mathbf{R}-\frac{1}{2} \operatorname{tr}\left[\operatorname{Curl}_{b}[\mathbf{R}] \cdot \mathbf{R}\right] 11,
$$

showing (2.16). Note the (more than formal?) coincidence with the relation $\mathfrak{G}=\mathfrak{R}-\frac{1}{2} \operatorname{tr}[\mathfrak{R}] \mathbb{1}$ between the symmetric Einstein curvature tensor $\mathfrak{G}$ and the symmetric Ricci curvature [13] tensor $\mathfrak{R}$ in the Einstein field equations of general relativity theory $[6,32]$. Taking matrix-norms on both sides of $\operatorname{Curl}_{b}[\mathbf{R}] \cdot \mathbf{R}=\widehat{\mathfrak{K}}-\operatorname{tr}[\widehat{\mathfrak{K}}] \mathbb{1}$ leads to

$$
\begin{aligned}
\left\|\operatorname{Curl}_{b}[\mathbf{R}] \cdot \mathbf{R}\right\|_{\mathbb{M}^{3 \times 3}}^{2} & =\|\widehat{\mathfrak{K}}-\operatorname{tr}[\widehat{\mathfrak{K}}] \mathbb{1}\|_{\mathbb{M}^{3 \times 3}}^{2}=\|\widehat{\mathfrak{K}}\|_{\mathbb{M}^{3 \times 3}}^{2}+(\operatorname{tr}[\widehat{\mathfrak{K}}])^{2} \\
& \geq\|\widehat{\mathfrak{K}}\|_{\mathbb{M}^{3 \times 3}}^{2}=\frac{1}{2}\|\mathrm{DR}\|_{\mathbb{R}^{27}}^{2}
\end{aligned}
$$

where we used (2.12) and $(4.4)_{2}$ to obtain the local inequalities, which is the claim. Note that for $n=2$ space dimensions we reproduce exactly the equality in (1.10).

Inspection of the proof shows that the estimate is already true for all orthogonal matrices. Counting equations in the cases of higher space dimensions suggests that the result remains true for arbitrary dimensions $n>3$. E.g. in $\mathrm{SO}(4)$ the corresponding gradient of the axial representation has 24 independent components (instead of 9 for $\mathrm{SO}(3)$ ) and the number of independent relations corresponding to taking the Curl is also 24. However, we have not looked at this case in detail.

\section{Note ADDED IN PROOF}

After submitting the paper the authors have been kindly made aware by A. Mielke, S. Müller and S. Conti of an elegant argument that applies to all space-dimensions and which also yields a constant independent of the dimension. We are grateful for this improvement and present their reasoning for the benefit of completeness of the development.

For $X \in C^{1}\left(\mathbb{R}^{n}, \mathbb{M}^{n \times n}\right)$ define the Curl in dimension $n \geq 3$ as the third order tensor

$$
(\operatorname{Curl} X(x))_{i j k}=\partial_{x_{k}} X^{i j}(x)-\partial_{x_{j}} X^{i k}(x)=X_{, k}^{i j}-X_{, j}^{i k}
$$

Let $W \in C^{1}\left(\mathbb{R}^{n}, \mathfrak{s o}(n)\right)$ be a skew-symmetric matrix in dimension $n \geq 3$. Then

$$
(\operatorname{Curl} W(x))_{i j k}=\partial_{x_{k}} W^{i j}(x)-\partial_{x_{j}} W^{i k}(x)=W_{, k}^{i j}-W_{, j}^{i k}=W_{, k}^{i j}+W_{, j}^{k i} .
$$


Using the skew-symmetry in the upper two indices of $W$ we may write

$$
\begin{aligned}
& \left(W_{, k}^{i j}-W_{, j}^{i k}\right)-\left(W_{, k}^{j i}-W_{, i}^{j k}\right)+\left(W_{, i}^{k j}-W_{, j}^{k i}\right) \\
& =W_{, k}^{i j}-W_{, j}^{i k}+W_{, k}^{i j}+W_{, i}^{j k}-W_{, i}^{j k}+W_{, j}^{i k}=2 W_{, k}^{i j},
\end{aligned}
$$

from which we infer the pointwise equivalence

$$
2 W_{, k}^{i j}(x)=(\operatorname{Curl} W(x))_{i j k}-(\operatorname{Curl} W(x))_{j i k}+(\operatorname{Curl} W(x))_{k j i},
$$

implying the control of all first partial derivatives of the skew-symmetric matrix $W$ in terms of a control of the Curl of this matrix, independent of the space-dimension $n$.

Consider now $R \in C^{1}\left(\mathbb{R}^{n}, \mathrm{SO}(n)\right)$ and expand in the neighbourhood of 0 :

$$
\begin{aligned}
R^{i j}(x) & =R^{i j}(0+x)=R^{i j}(0)+\sum_{k=1}^{n} \partial_{x_{k}} R^{i j}(0) x_{k}+o(x) \Rightarrow \\
R(0)^{T} R(x) & =\mathbb{1}_{n}+\sum_{k=1}^{n} \underbrace{\left[R(0)^{T} \partial_{x_{k}} R(0)\right]}_{\in \mathfrak{s o}(n)} x_{k}+o(x) .
\end{aligned}
$$

The first partial derivatives $\partial_{x_{k}} R(x)$ are still continuous, hence we may also write

$$
\begin{aligned}
\partial_{x_{k}} R(x) & =\partial_{x_{k}} R(0)+O(x) \Rightarrow \\
R(0)^{T} \partial_{x_{k}} R(x) & =\underbrace{R(0)^{T} \partial_{x_{k}} R(0)}_{\in \mathfrak{s o}(n)}+O(x) .
\end{aligned}
$$

Here and subsequently we let $o(x), O(x)$ denote the Landau symbols, respectively, i.e.,

$$
\lim _{x \rightarrow 0} \frac{o(x)}{x}=0, \quad \lim _{x \rightarrow 0} O(x)=0 .
$$

Since

$$
\begin{aligned}
\left(\operatorname{Curl}\left[R(0)^{T} R(x)\right]\right)_{i j k} & =\partial_{x_{k}}\left[R(0)^{T} R(x)\right]^{i j}-\partial_{x_{j}}\left[R(0)^{T} R(x)\right]^{i k} \\
& =R(0)^{T} \partial_{x_{k}} R(x)^{i j}-R(0)^{T} \partial_{x_{j}} R(x)^{i k} \\
& =R(0)^{T}\left[\partial_{x_{k}} R(x)^{i j}-\partial_{x_{j}} R(x)^{i k}\right] \\
& =R(0)^{T}(\operatorname{Curl} R(x))_{i j k}
\end{aligned}
$$

we conclude with (3.19) and (3.20) that

$$
\begin{aligned}
R(0)^{T}(\operatorname{Curl} R(x))_{i j k} & =\left(\operatorname{Curl}\left[R(0)^{T} R(x)\right]\right)_{i j k}=R(0)^{T}\left[\partial_{x_{k}} R(x)^{i j}-\partial_{x_{j}} R(x)^{i k}\right] \\
& =R(0)^{T}\left[\partial_{x_{k}} R(0)^{i j}-\partial_{x_{j}} R(0)^{i k}\right]+O(x),
\end{aligned}
$$

such that

$$
\left(\operatorname{Curl}\left[R(0)^{T} R(x)\right]\right)_{i j k}(x=0)=R(0)^{T}\left[\partial_{x_{k}} R(0)^{i j}-\partial_{x_{j}} R(0)^{i k}\right] .
$$


Define now

$$
W^{i j}(x)=\sum_{m=1}^{n}\left[R(0)^{T} \partial_{x_{m}} R(0)\right] x_{m} \in \mathfrak{s o}(n) \quad \Rightarrow \quad W_{, k}^{i j}(x)=R(0)^{T} \partial_{x_{k}} R(0) .
$$

Thus we have

$$
R(0)^{T} R(x)=\mathbb{1}_{n}+\sum_{m=1}^{n}\left[R(0)^{T} \partial_{x_{m}} R(0)\right] x_{m}+o(x)=\mathbb{1}_{n}+W^{i j}(x)+o(x)
$$

and

$$
(\operatorname{Curl} W)_{j i k}(0)=W_{, k}^{i j}(0)-W_{, j}^{i k}(0)=R(0)^{T}\left[\partial_{x_{k}} R(0)^{i j}-\partial_{x_{j}} R(0)^{i k}\right]
$$

This yields with (3.19) and (3.25)

$$
\begin{aligned}
2 \partial_{x_{k}}\left[R(0)^{T} R(x)\right]= & 2 W_{, k}^{i j}(0)+O(x) \\
= & (\operatorname{Curl} W)_{i j k}(0)-(\operatorname{Curl} W)_{j i k}(0)+(\operatorname{Curl} W)_{k j i}(0)+O(x) \\
= & \left(\operatorname{Curl}\left[R(0)^{T} R(x)\right]\right)_{i j k}(x=0)-\left(\operatorname{Curl}\left[R(0)^{T} R(x)\right]\right)_{j i k}(x=0) \\
& \quad+\left(\operatorname{Curl}\left[R(0)^{T} R(x)\right]\right)_{k j i}(x=0)+O(x) \\
= & R(0)^{T}\left[(\operatorname{Curl} R(x))_{i j k}(x=0)-(\operatorname{Curl} R(x))_{j i k}(x=0)\right. \\
& \left.\quad+(\operatorname{Curl} R(x))_{k j i}(x=0)+O(x)\right],
\end{aligned}
$$

where we have used (3.20) and (3.17). Thus

$$
\begin{aligned}
2 R(0)^{T} \partial_{x_{k}}[R(x)]=R(0)^{T}[ & (\operatorname{Curl} R(x))_{i j k}(x=0)-(\operatorname{Curl} R(x))_{j i k}(x=0) \\
+ & \left.(\operatorname{Curl} R(x))_{k j i}(x=0)+O(x)\right]
\end{aligned}
$$

Hence, setting $x=0$ we obtain the equivalence

$$
2 \partial_{x_{k}} R(0)=\left[(\operatorname{Curl} R)_{i j k}(0)-(\operatorname{Curl} R)_{j i k}(0)+(\operatorname{Curl} R)_{k j i}(0)\right]
$$

Since $R(0)$ is arbitrary this proves the claim. From (3.28) one obtains, without detailed indicial calculation, using only the Cauchy-Schwarz inequality

$$
\forall R \in C^{1}\left(\mathbb{R}^{n}, \mathrm{SO}(n)\right): \quad\|\operatorname{Curl} R\|_{\mathbb{R}^{n^{3}}}^{2} \geq \frac{4}{9}\|\mathrm{D} R\|_{\mathbb{R}^{n^{3}}}^{2},
$$

with a constant independent of the space-dimension.

Acknowledgements. This work was written in the academic year WS05/06 while P.N. held a non-permanent position at FB Mathematik, Universität Duisburg-Essen, Campus Essen. P.N. would like to thank N. Weck, K.J. Witsch, A. Klawonn and J. Schröder for their kind hospitality. P.N has also profited from many helpful discussions over the last years with B. Svendsen on dislocation theory. He is also grateful to A. Mielke for stimulating exchange on the proper formulation of dislocation based plasticity theory. I.M. is grateful to W. Wagner for supervision and support. The result in this paper has been announced at the GAMM-conference, March 2006, Berlin. 


\section{Appendix: The ClassicAl Linearized RELATIONS}

Interpreting Kröners [15-18] development in our notation, the dislocation density tensor of the linearized theory $\alpha$ is defined as $\alpha=-\operatorname{Curl}_{\sharp}\left[\varepsilon^{\mathrm{p}}\right]$. Thus one may write

$$
-\alpha:=\operatorname{Curl}_{\sharp}\left[\varepsilon^{\mathrm{p}}\right]=\underbrace{\operatorname{Curl}_{\sharp}\left[\operatorname{sym}\left[\varepsilon^{\mathrm{p}}\right]\right]}_{\text {plastic strain curl-tensor }}+\underbrace{\operatorname{Cur} l_{\sharp}\left[\operatorname{skew}\left[\varepsilon^{\mathrm{p}}\right]\right]}_{\text {plastic curvature tensor }} .
$$

Kröner frequently highlighted the relation between Cartan's torsion tensor [3] of the crystal connection and the dislocation density tensor $\alpha$ [17], p. 292, equation (38), [18], p. 103, on the one hand and Nye's curvature tensor [17], p. 292, equation (39), on the other hand. According to [18] the relation between Cartan's torsion and dislocation density tensor has first been established in [2,14]. In the nonlinear theory Kröner writes [17]

$$
\alpha_{m l k}:=\frac{1}{2}\left(\partial_{m} F_{k l}^{\mathrm{p}}-\partial_{l} F_{k m}^{\mathrm{p}}\right)=\frac{1}{2} \epsilon_{m l n}\left(\operatorname{Curl}_{b}\left[F^{\mathrm{p}}\right]\right)_{n k}=\frac{1}{2} \epsilon_{m l n}\left(\operatorname{Curl}_{\sharp}\left[F^{\mathrm{p}}\right]\right)_{k n},
$$

where $\alpha_{m l k}$ is Kröners third order dislocation density tensor and $F^{\mathrm{p}}=11+\varepsilon^{\mathrm{p}}$ is understood. Cartan's torsion tensor is the anti-symmetric part $\Gamma_{[\mathrm{ml}] k}$ in the first two indices of the (non-symmetric hence non-affine) crystal connection $\Gamma_{m l k}$ defined implicitly through

$$
\partial_{m}\left(F^{\mathrm{p}}(x) \cdot \mathbf{e}_{l}\right)=\Gamma_{m l k}(x) \cdot\left(F^{\mathrm{p}}(x) \cdot \mathbf{e}_{k}\right),
$$

and the relation is $\Gamma_{[m l] k}=\alpha_{m l k}$. To proceed let us note the useful algebraic relations

$$
\begin{aligned}
& X-\operatorname{tr}[X] \mathbb{1}_{n}=Y \Leftrightarrow X=Y-\frac{1}{n-1} \operatorname{tr}[Y] \mathbb{1}_{n}, \\
& X \in \mathbb{M}^{n \times n}: \quad\left\|X-\operatorname{tr}[X] \mathbb{1}_{n}\right\|_{\mathbb{M}^{n \times n}}^{2}=\|X\|_{\mathbb{M}^{n \times n}}^{2}+(n-2)(\operatorname{tr}[X])^{2}, \quad n \geq 2,
\end{aligned}
$$

which can be seen by straightforward calculation.

In [26] Nye considered the case of a plastically bent single crystal with small angle tilt grain boundaries which are in a state of polygonisation, assumed to be representing a low energy configuration of the crystal. There, the plastic distortion $\varepsilon^{\mathrm{p}}$ consists only of skew-symmetric parts which lead to infinitesimal plastic rotations of the lattice. This means, there is no attendent plastic strain $\operatorname{sym}\left[\varepsilon^{\mathrm{p}}\right]=0$. The skew-symmetric $\varepsilon^{\mathrm{p}}$ locally rotates the lattice (compatible rotations in the sense of Kröner [17]) without causing macroscopic (long range elastic lattice) stresses. Nye defines his curvature tensor in the following way [26]

$$
\kappa_{\text {Nye }}:=\nabla \operatorname{axl}\left[\operatorname{skew}\left[\varepsilon^{\mathrm{p}}\right]\right]=\operatorname{Grad}\left[\operatorname{axl}\left[\operatorname{skew}\left[\varepsilon^{\mathrm{p}}\right]\right]\right]=-\frac{1}{2} \epsilon_{i k l} \varepsilon_{k l, j}^{\mathrm{p}} \mathbf{e}_{i} \otimes \mathbf{e}_{j}
$$

such that $\kappa_{\text {Nye }}$ measures the infinitesimal change in orientation of neighboured lattices. With respect to Nye's curvature compare also with [7].

The relation between Nyes curvature $\kappa_{\text {Nye }}$ and Kröners definition of the dislocation density tensor of the linearized theory for the restricted case of a skew-symmetric $\varepsilon^{\mathrm{p}}$ is

$$
\boldsymbol{\alpha}=\boldsymbol{\kappa}_{\mathrm{Nye}}^{T}-\operatorname{tr}\left[\boldsymbol{\kappa}_{\mathrm{Nye}}^{T}\right] \mathbb{1}, \quad \boldsymbol{\kappa}_{\mathrm{Nye}}=\boldsymbol{\alpha}^{T}-\frac{1}{2} \operatorname{tr}\left[\boldsymbol{\alpha}^{T}\right] 11
$$


This linear relation (4.6) is well known [18], p. 103, and is easily established (called Nye's relation), cf. [4], equation (7.10). We write

$$
\begin{aligned}
& \boldsymbol{\alpha}=\boldsymbol{\kappa}_{\mathrm{Nye}}^{T}-\operatorname{tr}\left[\boldsymbol{\kappa}_{\mathrm{Nye}}^{T}\right] \mathbb{1}=\left(-\frac{1}{2} \epsilon_{j k l} \varepsilon_{k l, i}^{\mathrm{p}}+\frac{1}{2} \epsilon_{a k l} \varepsilon^{\mathrm{p}}{ }_{k l, a} \delta_{i j}\right) \mathbf{e}_{i} \otimes \mathbf{e}_{j} \\
& =-\frac{1}{2}\left(\delta_{j h} \epsilon_{h k l} \varepsilon^{\mathrm{p}} k l, a \delta_{a i}-\delta_{i j} \delta_{h a} \epsilon_{h k l} \varepsilon^{\mathrm{p}}{ }_{k l, a}\right) \mathbf{e}_{i} \otimes \mathbf{e}_{j} \\
& =-\frac{1}{2}\left(\delta_{j h} \delta_{a i}-\delta_{i j} \delta_{h a}\right) \epsilon_{h k l} \varepsilon^{\mathrm{p}} k l, a \mathbf{e}_{i} \otimes \mathbf{e}_{j}=-\frac{1}{2} \epsilon_{j a b} \epsilon_{h i b} \epsilon_{h k l} \varepsilon^{\mathrm{p}}{ }_{k l, a} \mathbf{e}_{i} \otimes \mathbf{e}_{j} \\
& =-\epsilon_{j a b} \partial_{a}\left(\frac{1}{2} \epsilon_{h i b} \epsilon_{h k l} \varepsilon^{\mathrm{p}}{ }_{k l}\right) \mathbf{e}_{i} \otimes \mathbf{e}_{j}=-\partial_{a} \epsilon_{a b j}\left(\left(\operatorname{skew}\left[\varepsilon^{\mathrm{p}}\right]\right)^{T}\right)_{b i} \mathbf{e}_{i} \otimes \mathbf{e}_{j} \\
& =-\left(\nabla \times\left(\operatorname{skew}\left[\varepsilon^{\mathrm{p}}\right]\right)^{T}\right)_{j i} \mathbf{e}_{i} \otimes \mathbf{e}_{j}=-\left(\operatorname{Curl}_{b}\left[\operatorname{skew}\left[\varepsilon^{\mathrm{p}}\right]\right]\right)_{j i} \mathbf{e}_{i} \otimes \mathbf{e}_{j} \\
& \left.=-\left(\operatorname{Curl} l_{b}\left[\operatorname{skew}\left[\varepsilon^{\mathrm{p}}\right]\right]\right)_{i j}^{T} \mathbf{e}_{i} \otimes \mathbf{e}_{j}=-(\operatorname{Curl}]_{\sharp}\left[\operatorname{skew}\left[\varepsilon^{\mathrm{p}}\right]\right]\right)_{i j} \mathbf{e}_{i} \otimes \mathbf{e}_{j}=\boldsymbol{\alpha} .
\end{aligned}
$$

This, together with (4.5) and (4.4) $)_{1}$ or $(4.6)_{2}$ implies that for all $A \in \mathfrak{s o}(3)$ we have

$$
\nabla \operatorname{axl}[A]=-\left(\operatorname{Curl}_{\sharp}[A]\right)^{T}+\frac{1}{2} \operatorname{tr}\left[\left(\operatorname{Curl}_{\sharp}[A]\right)^{T}\right] 11 .
$$

Inverting this relation again with $(4.4)_{1}$ and taking the norm on both sides leads with $(4.4)_{2}$ to

$$
\left\|\operatorname{Curl}_{\sharp}[A]\right\|_{\mathbb{M}^{3 \times 3}}^{2} \geq\|\nabla \operatorname{axl}[A]\|_{\mathbb{M}^{3 \times 3}}^{2}=\frac{1}{2}\|\mathrm{D} A\|_{\mathbb{R}^{27}}^{2}
$$

\section{REFERENCES}

[1] S. Aubry and M. Ortiz, The mechanics of deformation-induced subgrain-dislocation structures in metallic crystals at large strains. Proc. R. Soc. Lond., Ser. A, Math. Phys. Eng. Sci. 459 (2003) 3131-3158.

[2] B.A. Bilby, R. Bullough and E. Smith, Continuous distributions of dislocations: a new application of the methods of nonriemannian geometry. Proc. Roy. Soc. London, Ser. A 231 (1955) 263-273.

[3] E. Cartan, Leçons sur la géometrie des espaces de Riemann. Gauthier-Villars, Paris (1928).

[4] P. Cermelli and M.E. Gurtin, On the characterization of geometrically necessary dislocations in finite plasticity. J. Mech. Phys. Solids 49 (2001) 1539-1568.

[5] S. Conti and M. Ortiz, Dislocation microstructures and the effective behavior of single crystals. Arch. Rat. Mech. Anal. 176 (2005) 103-147.

[6] A. Einstein, Relativity: The Special and General Theory. Crown, New-York (1961).

[7] J.D. Eshelby, The continuum theory of lattice defects, volume III of Solid state Physics. Academic Press, New-York (1956).

[8] G. Friesecke, R.D. James and S. Müller, A theorem on geometric rigidity and the derivation of nonlinear plate theory from three-dimensional elasticity. Comm. Pure Appl. Math. 55 (2002) 1461-1506.

[9] M.E. Gurtin, An Introduction to Continuum Mechanics, Mathematics in Science and Engineering 158. Academic Press, London, 1st edn. (1981).

[10] M.E. Gurtin, On the plasticity of single crystals: free energy, microforces, plastic-strain gradients. J. Mech. Phys. Solids $\mathbf{4 8}$ (2000) 989-1036.

[11] J.P. Hirth and J. Lothe, Theory of Dislocations. McGraw-Hill, New-York (1968).

[12] F. John, Rotation and strain. Comm. Pure Appl. Math. 14 (1961) 391-413.

[13] J. Jost, Riemannian Geometry. Springer-Verlag (2002).

[14] K. Kondo, Geometry of elastic deformation and incompatibility, in Memoirs of the Unifying Study of the Basic Problems in Engineering Science by Means of Geometry, volume 1, Division C, K. Kondo Ed., Gakujutsu Bunken Fukyo-Kai (1955) 361-373.

[15] E. Kröner, Der fundamentale Zusammenhang zwischen Versetzungsdichte und Spannungsfunktion. Z. Phys. 142 (1955) 463475.

[16] E. Kröner, Kontinuumstheorie der Versetzungen und Eigenspannungen, Ergebnisse der Angewandten Mathematik 5. Springer, Berlin (1958).

[17] E. Kröner, Allgemeine Kontinuumstheorie der Versetzungen und Eigenspannungen. Arch. Rat. Mech. Anal. 4 (1960) $273-334$.

[18] E. Kröner and A. Seeger, Nichtlineare Elastizitätstheorie der Versetzungen und Eigenspannungen. Arch. Rat. Mech. Anal. 3 (1959) 97-119. 
[19] D. Kuhlmann-Wilsdorf, Theory of plastic deformation: properties of low energy dislocation structures. Mat. Sci. Eng. A113 (1989) 1.

[20] E.H. Lee, Elastic-plastic deformation at finite strain. J. Appl. Mech. 36 (1969) 1-6.

[21] A. Mielke and S. Müller, Lower semi-continuity and existence of minimizers in incremental finite-strain elastoplasticity. ZAMM 86 (2006) 233-250.

[22] T. Mura, Micromechanics of defects in solids. Kluwer Academic Publishers, Boston (1987).

[23] F.R.N. Nabarro, Theory of crystal dislocations. Oxford University Press, Oxford (1967).

[24] J. Necas and I. Hlavacek, Mathematical theory of elastic and elastico-plastic bodies: An introduction. Elsevier, Amsterdam (1981).

[25] P. Neff, On Korn's first inequality with nonconstant coefficients. Proc. Roy. Soc. Edinb. A 132 (2002) $221-243$.

[26] J.F. Nye, Some geometrical relations in dislocated crystals. Acta Metall. 1 (1953) 153-162.

[27] M. Ortiz and E.A. Repetto, Nonconvex energy minimization and dislocation structures in ductile single crystals. J. Mech. Phys. Solids 47 (1999) 397-462.

[28] M. Ortiz, E.A. Repetto and L. Stainier, A theory of subgrain dislocation structures. J. Mech. Phys. Solids 48 (2000) $2077-2114$.

[29] G.P. Parry and M. Silhavy, Elastic scalar invariants in the theory of defective crystals. R. Soc. Lond. Proc., Ser. A, Math. Phys. Eng. Sci. 455 (1999) 4333-4346.

[30] Yu.G. Reshetnyak, Liouville's theorem on conformal mappings for minimal regularity assumptions. Siberian Math. J. 8 (1967) 631-653.

[31] B. Svendsen, Continuum thermodynamic models for crystal plasticity including the effects of geometrically necessary dislocations. J. Mech. Phys. Solids 50 (2002) 1297-1329.

[32] R.M. Wald, General Relativity. University of Chicago Press, Chicago (1984). 